\title{
Regulated degradation of the APC coactivator Cdc20
}

Jonathan A Robbins, Frederick R Cross ${ }^{*}$

\begin{abstract}
Background: Cdc20 is a highly conserved activator of the anaphase-promoting complex (APC), promoting cellcycle-regulated ubiquitination and proteolysis of a number of critical cell-cycle-regulatory targets including securin and mitotic cyclins. APC-Cdc20 activity is tightly regulated, and this regulation is likely important for accurate cell cycle control. One significant component of $\mathrm{Cdc} 20$ regulation is thought to be $\mathrm{Cdc} 20$ proteolysis. However, published literature suggests different mechanisms and requirements for $\mathrm{Cdc20}$ proteolysis. The degree to which Cdc20 proteolysis is cell-cycle regulated, the dependence of Cdc20 proteolysis on Cdc20 destruction boxes (recognition sequences for APC-mediated ubiqutination, either by Cdc20 or by the related Cdh1 APC activator), and the need for APC itself for Cdc20 proteolysis all have been disputed to varying extents. In animals, Cdc20 proteolysis is thought to be mediated by Cdh1, contributing an intrinsic order of APC activation by Cdc20 and then by Cdh1. One report suggests a Cdh1 requirement for Cdc20 proteolysis in budding yeast; this idea has not been tested further.
\end{abstract}

Results: We characterized Cdc20 proteolysis using Cdc20 expressed from its endogenous locus; previous studies generally employed strongly overexpressed Cdc20, which can cause significant artifacts. We analyzed Cdc20 proteolysis with or without mutations in previously identified destruction box sequences, using varying methods of cell cycle synchronization, and in the presence or absence of Cdh1. Cdc20 instability is only partially dependent on destruction boxes. A much stronger dependence on Cdh1 for Cdc20 proteolysis was observed, but Cdh1independent proteolysis was also clearly observed. Cdc20 proteolysis independent of both destruction boxes and Cdh1 was especially detectable around the G1/S transition; Cdh1-dependent proteolysis was most notable in late mitosis and G1.

Conclusions: Cdc20 proteolysis is under complex control, with different systems operating at different points in the cell cycle. This complexity is likely to explain apparent conflicts in previously published literature on this subject. A major mode of control of Cdc20 proteolysis occurs in late mitosis/early G1 and is Cdh1-dependent, as in animal cells; this mode may contribute to the known sequential activation of the APC by Cdc20 followed by Cdh1. An independent mode of Cdc20 proteolysis, independent of destruction boxes and Cdh1, occurs at G1/S; we do not know the mechanism or function of this mode of proteolysis, but speculate that it may contribute to sharpening and restricting activation of APC-Cdc20 to early mitosis.

\section{Background}

The oscillation of cyclin dependent kinase (CDK) activity lies at the heart of the cell cycle, serving to coordinate the events of the cell cycle in a temporally appropriate manner. CDK activity is dependent upon CDK binding to a partner cyclin [1]; to exit from mitosis, the CDK activity of the mitotic B-type cyclins must

\footnotetext{
* Correspondence: fred.r.cross@gmail.com
Laboratory of Yeast Molecular Genetics, The Rockefeller University, New York,

* Correspondence: fred.r.cross@gmail.com NY, USA
}

(c) 2010 Robbins and Cross; licensee BioMed Central Ltd. This is an Open Access article distributed under the terms of the Creative Commons Attribution License (http://creativecommons.org/licenses/by/2.0), which permits unrestricted use, distribution, and reproduction in any medium, provided the original work is properly cited.
The anaphase-promoting com enase responsible for the destruction of cyclins at the end of mitosis: the cell cycle ends in highly efficient and specific protein destruction orchestrated by the APC, which mediates the sequential degradation of cyclins and other relevant cell cycle proteins and machinery $[2,3]$.

The APC is a large ubiquitin E3 ligase comprised of at least 13 proteins, and functions in coordination with

\section{Biomed Central}


two homologous mitotic coactivators, Cdc20p and Cdh1 [4-8]. The APC and both coactivators are conserved throughout eukaryotic evolution. The APC is active only from anaphase onset through the subsequent G1, although the core complex is present throughout the cell cycle. The conserved coactivators Cdc 20 and Cdh1 provide regulation of timing and specificity. APC-Cdc20 begins B-type cyclin degradation and APC-Cdh1 continues it through mitosis and into the ensuing G1 [5,9-13].

A major basis for this difference in timing is differential regulation of APC-Cdc20 and APC-Cdh1 by cyclinCDK activity. APC-Cdc20 is active at high CDK levels, with Cdc20 binding preferentially to CDK-phosphorylated APC [14,15]. Cdc20 itself is an unstable protein, accumulating late in the cell cycle, followed by mitotic degradation [16-18]. As B-type cyclin levels decline and the Cdc14 phosphatase (at least in budding yeast) is released from a nucleolar sequestration, the balance between CDK activity and phosphatase activity shifts such that Cdh1 is dephosphorylated on at least some of its 11 CDK sites, which collectively serve to inhibit Cdh1 function [10]. The second wave of APC-mediated degradation then ensues, dependent on dephosphorylated Cdh1. This activity is responsible for continued mitotic cyclin degradation through G1, until Cdh1 inactivation in the succeeding cell cycle [19].

In addition to these temporal differences, Cdc20 and Cdh1 likely have intrinsically different substrate specificities, although they both contribute to mitotic cyclin degradation. Cdc20 promotes Pds1 proteolysis, an anaphase inhibitor that prevents cleavage of cohesin, the protein keeping sister chromatids attached $[11,20]$. APC-Cdh1 seems ineffective at promoting Pds1 degradation, but promotes degradation of several spindle proteins and perhaps Cdc20 itself [5,10,16,21-24]. This ordering is logical: the earlier APC-Cdc20 wave will promote anaphase and initial mitotic cyclin proteolysis, promoting APC-Cdh1 activation; APC-Cdh1 then completes mitotic cyclin proteolysis, allowing cytokinesis and other events of mitotic exit, removes Cdc20 to reset the system to G1, and contributes to spindle disassembly by proteolysis of spindle components. This ordering could help ensure that anaphase precedes cytokinesis and spindle disassembly.

Specific motifs in substrate proteins target them for APC-mediated ubiquitination: the destruction box (consensus RxxL) [25], recognized by Cdc20 and Cdh1; the KEN box, which may be more specific for Cdh1 [26] (but see [27]), and the CRY box Cdh1 recognition sequence [28]. Thus $C \mathrm{dh} 1$ recognizes unique motifs that Cdc20 does not; in contrast, there are no known Cdc20specific targeting sequences, although Cdc20 specific substrates exist.
$C D C 20$ is essential for cell viability, and its absence results in an arrest with unseparated sister chromatids and high Clb2 levels $[16,29]$. Deletion of the APCCdc20 target PDS1 (securin) allows $c d c 20$ cells to undergo anaphase $[16,29]$. Further deletion of CLB5 results in a viable $c d c 20$ pds 1 clb5 triple mutant, capable of carrying out all essential cell-cycle functions [11]. This defines two critical targets of Cdc20; consistently, both have been reported to be poor APC-Cdh1 substrates $[5,6]$.

Temporal separation of APC-Cdc20 and APC-Cdh1 activity is thought to promote ordering of degradation of APC substrates. Not only must Cdh1 activity be restrained until mitotic exit, but it is likely that $\mathrm{Cdc} 20$ must be inactivated for the subsequent cell cycle. Inability to inactivate $\mathrm{Cdc} 20$ would impede securin accumulation, impairing separase regulation, and constitutive Cdc20 could also block accumulation of the major Sphase cyclin Clb5.

Three mechanisms are known to contribute to Cdc20 inactivation: the dephosphorylation of the APC $[14,15]$, transcriptional shutoff [17], and the destruction of Cdc20 itself [16-18,22,30]. Cdc20 has two destruction boxes thought to target it for destruction [16]. It has been argued based on the stabilization of overexpressed alleles deleted for the region containing destruction boxes that both destruction boxes contribute to Cdc20 degradation in G1 $[16,17]$. One study also found residual APC-dependent but destruction box independent Cdc20 instability throughout the entire cell cycle [17]. Another study argued that degradation of $\mathrm{Cdc} 20$ was dependent only on the first destruction box [30], and was cellcycle-regulated (highest in G1), but was independent of APC activity. These substantially contradictory results hamper understanding of this potentially important regulatory event.

\section{Results}

\section{The role of the Cdc20 destruction boxes}

We sought to test the consequences of removing the individual Cdc20 destruction boxes, in the context of the endogenous locus and promoter, using the Nterminally myc-epitope-tagged construct described by [16]. The destruction box mutations were alterations of the RxxL consensus site to AxxA. CDC20-db1 lacks the first destruction box, $C D C 20-d b 2$ the second, and $C D C 20-d b 3$ both (nomenclature of [16]). These alleles were functional, as they replaced the endogenous copy of $C D C 20$, which is an essential gene. Additionally, they were also viable in the absence of $C D H 1$, a genetic background known to be sensitive to hypoactive APC-Cdc20 [31]. Cells were synchronized in alpha-factor and released, with Myc-Cdc20 accumulation monitored by western blotting against Myc (Figure 1). Wild-type 


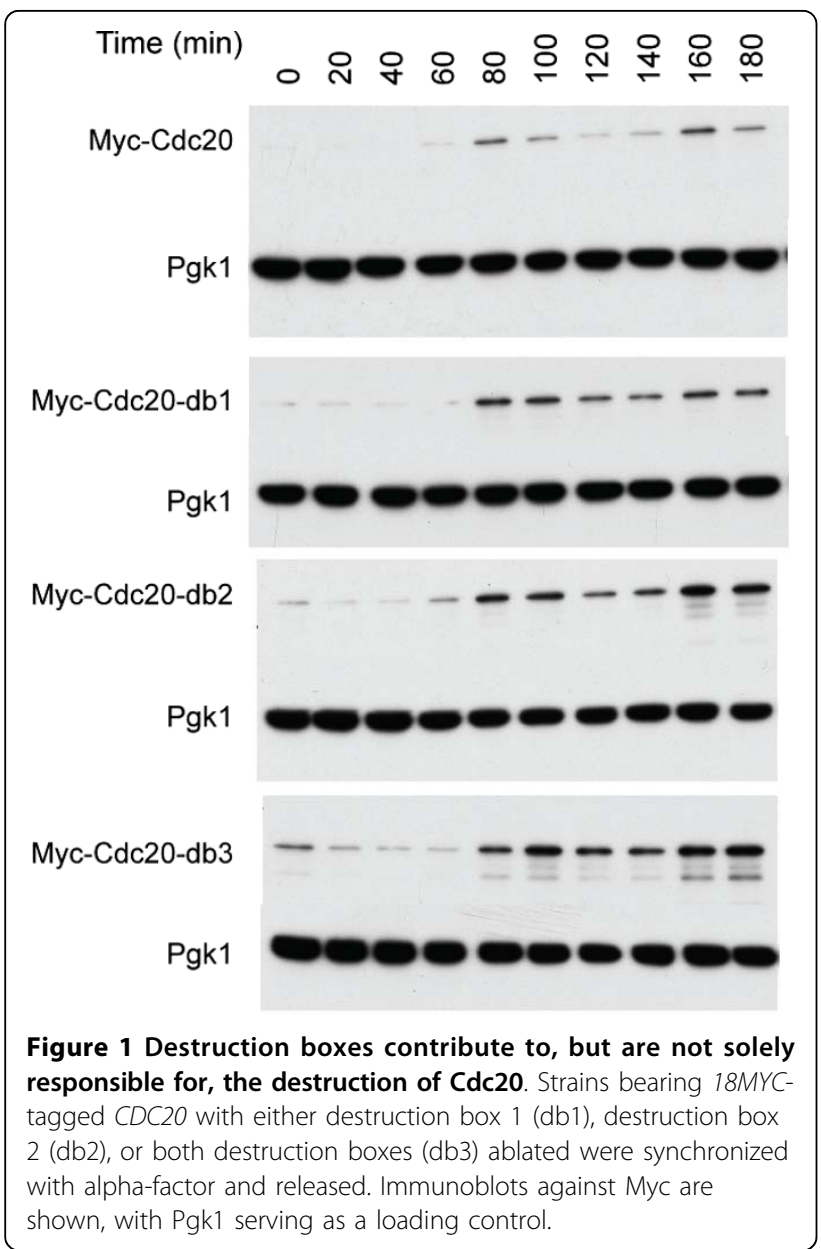

Cdc20 was completely gone at the alpha-factor block; this clearance required the two destruction boxes (Figure 2A). Upon release from alpha-factor, Cdc20 accumulated strikingly, with a second drop and reaccumulation detectable (presumably due to mitosis and cell cycle reentry). While the $\mathrm{db}$ mutations resulted in increased Cdc20 levels and less efficient clearance in mitosis, even $\mathrm{Cdc} 20$ - $\mathrm{db} 3$ retained a pattern of accumulation generally similar to wild-type.

We noted a reproducible decrease in the db-mutated Cdc20 proteins upon release of the alpha-factor block. (A similar effect was detectable upon long exposure with wild-type Cdc20, despite the extremely low initial levels; data not shown.) $C D C 20$ transcription is low at alpha-factor arrest, and only increases late in the cell cycle [32]; thus, the decline in Cdc20 levels shortly after release from the alpha-factor block is probably not the consequence of transcriptional downregulation. Rather, Cdc20 appears to be actively destroyed by some process as cells progress through G1 into S. This degradation is destruction box-independent, and is unlikely to be APC-Cdh1 mediated, as Cdh1 activity is very high in alpha-factor-blocked cells and declines to negligible levels upon release.

Overexpression of $C D C 20$ lacking destruction boxes has been reported to interfere with S-phase progression (perhaps due to APC-Cdc20 ubiquitination of the Sphase cyclin Clb5) [11]. However, with CDC20-db3 expressed at endogenous levels we observe at most minor effects on DNA replication kinetics (Figure 2B).

\section{Overexpression of $\mathrm{CDH} 1$ partially reduces $\mathrm{Cdc} 20$ levels}

APC-Cdh1 activity has been reported to restrain Cdc20 accumulation until early S-phase [22]. Cdc20 was nearly completely removed in alpha-factor-blocked cells, even in the absence of its destruction boxes. Alpha-factorblocked cells contain high APC-Cdh1 activity, which could account for Cdc20 clearance in these cells. Therefore, we tested whether Cdh1 overexpression in cycling cells is able to clear Cdc20 expressed from the endogenous locus. Overexpressed $C D H 1$ had little effect on Cdc20 levels, but this could be due to efficient Cdk phosphorylation and inactivation of Cdh1 (Figure 3). CDH1-m11, lacking Cdk sites, reduces the level of Cdc20 significantly; however, this effect was incomplete even after two hours of CDH1-m11 induction (Figure 3), a time long enough for Cdh1-m11 to induce cell cycle arrest with a hyperpolarized morphology characteristic of an absence of B-type cyclins (data not shown). This induction results in significant overexpression as compared to expression from the endogenous promoter ([10]; data not shown). It is unclear why overexpressed constitutively active Cdh1 is unable to completely clear Cdc20, which is in contrast to the complete clearance seen in alpha-factor blocked cells, or in CDH1-m11 (exact gene replacement) cells released from alpha-factor and accumulating at the CDH1-m11 block[33]. As this is an initially asynchronous culture, and CDH1-m11 expressing cells arrest in the first cycle, it possible that during this arrest Cdc20 is a poor Cdh1 substrate, perhaps owing to posttranslational modifications, association with the APC or spindle checkpoint proteins, or spatial sequestration of $\mathrm{Cdc} 20$. It is also possible that $C D C 20$ transcription at this block is sufficient to reach a steady-state equilibrium.

\section{Cdc20 levels are increased but still cell cycle regulated in} the absence of $\mathrm{CDH} 1$

To examine the effect of endogenous Cdh1 on Cdc20 levels, we used centrifugal elutriation to separate cycling $c d h 1$ and wild-type cells into different cell cycle fractions based on cell size (we were unable to use alpha-factor synchronization for this experiment because $c d h 1$ cells do not arrest properly in response to alpha-factor [5]. The use of size fractionation followed by direct analysis of differentsized cell fractions has been validated previously for 

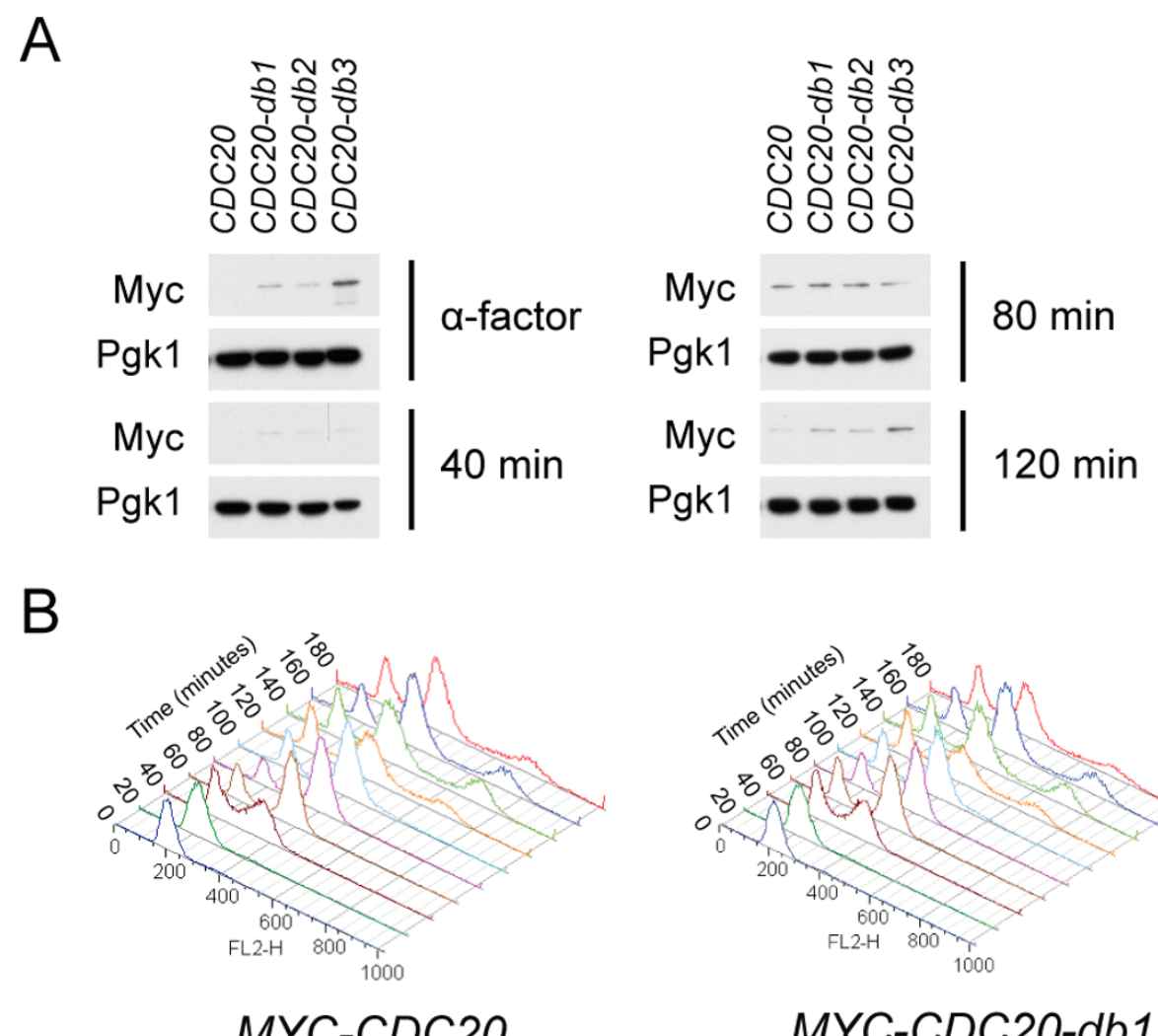

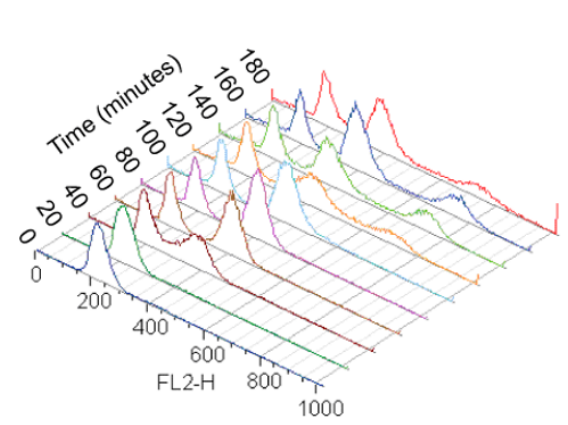

MYC-CDC20-db2

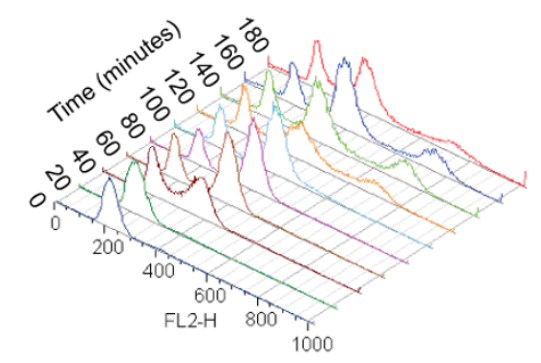

MYC-CDC20-db1

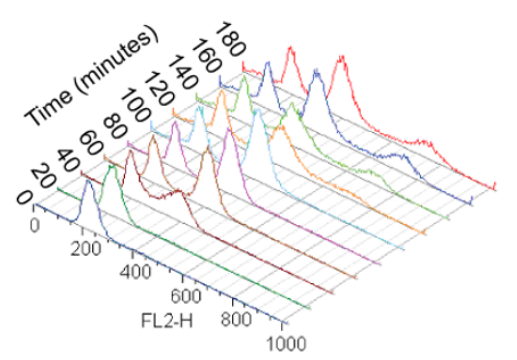

MYC-CDC20-db3

Figure 2 Ablation of destruction boxes particularly stabilizes Cdc20 in alpha-factor, but does not affect DNA replication kinetics. A Samples from Figure 1 for each CDC20 allele at indicated timepoints were loaded next to one another on the same gel and blotted against. B FACS analysis of DNA content from samples taken in parallel with those from Figure 1.

wild-type and $c d h 1$ mutants [34]). $c d h 1$ cells have higher levels of Cdc20 than $C D H 1$ cells, particularly in G1; however, Cdc20 declines to a low level in S-phase $c d h 1$ cells (Figure 4), before increasing later in the cell cycle. Thus, Cdh1 may be responsible for clearance of Cdc20 in G1, but there appear to be Cdh1-independent mechanisms of Cdc20 clearance operating later in the cell cycle. These observations are consistent with those made above with alpha-factor synchronized $C D C 20-d b 3$ cells, and with CDH1-m11-overexpressing cells.
Inducible Cdc20 is degraded by both Cdh1-dependent and independent mechanisms

To pursue Cdh1-dependent and-independent mechanisms of Cdc 20 proteolysis, independent of transcriptional regulation of $C D C 20$, we employed an inducible MET3-HA-CDC20 replacing the endogenous copy of $C D C 20$. Depletion of $\mathrm{Cdc} 20$ in this strain by methionine addition results in a reversible metaphase arrest. MET3$H A-C D C 20 C D H 1$ and MET3-HA-CDC2O cdh1 strains were grown to log phase in medium lacking methionine. 


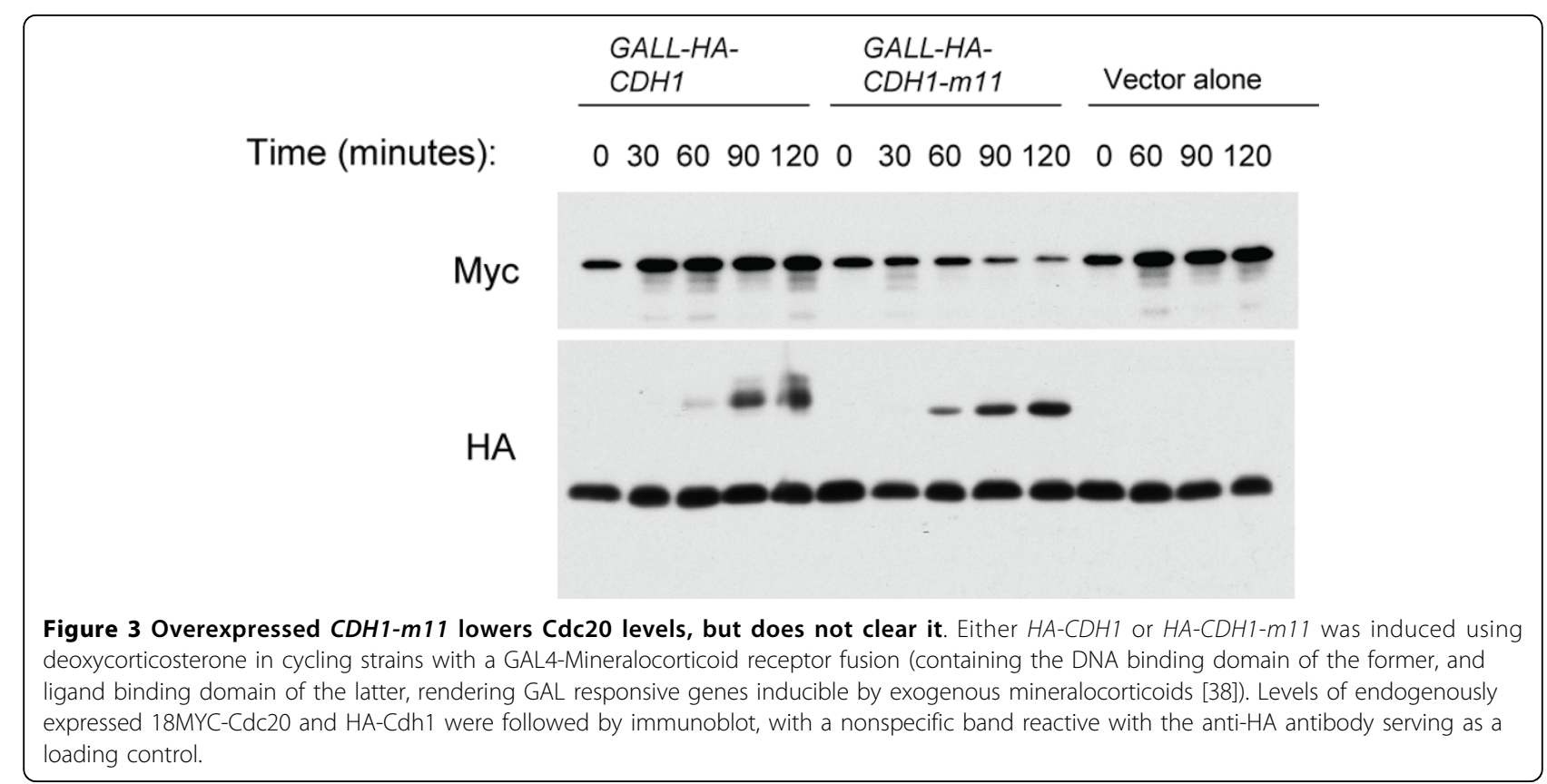

Methionine was then added; 30 minutes later, neither strain contained detectable Cdc20, once again confirming the existence of efficient Cdh1-independent mechanisms of Cdc20 degradation (Figure 5). Upon induction of $C D C 20$ to release the metaphase block, Cdc20 levels increase much more in a $c d h 1$ than in a $C D H 1$ background, confirming the ability of Cdh1 to promote
Cdc20 degradation during mitotic exit. However, after a short additional time Cdc20 levels decrease substantially in the $c d h 1$ cells as well (although the levels remain higher than in CDH1 cells) (Figure 5).

These results confirm the existence of separable Cdh1-dependent and-independent mechanisms of Cdc20 proteolysis.

A

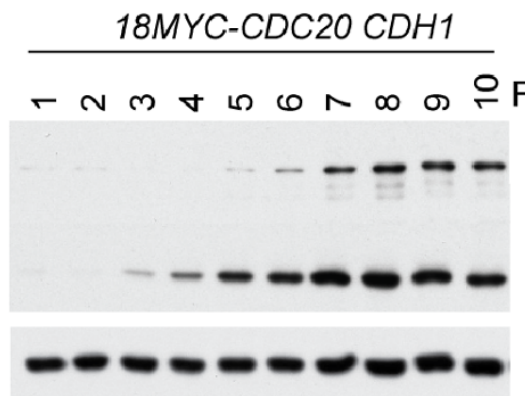

B

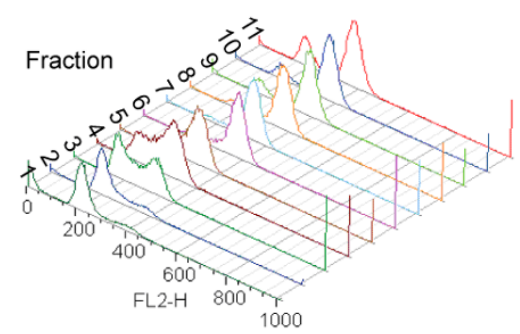

$18 M Y C-C D C 20 \operatorname{cdh} 1 \Delta$
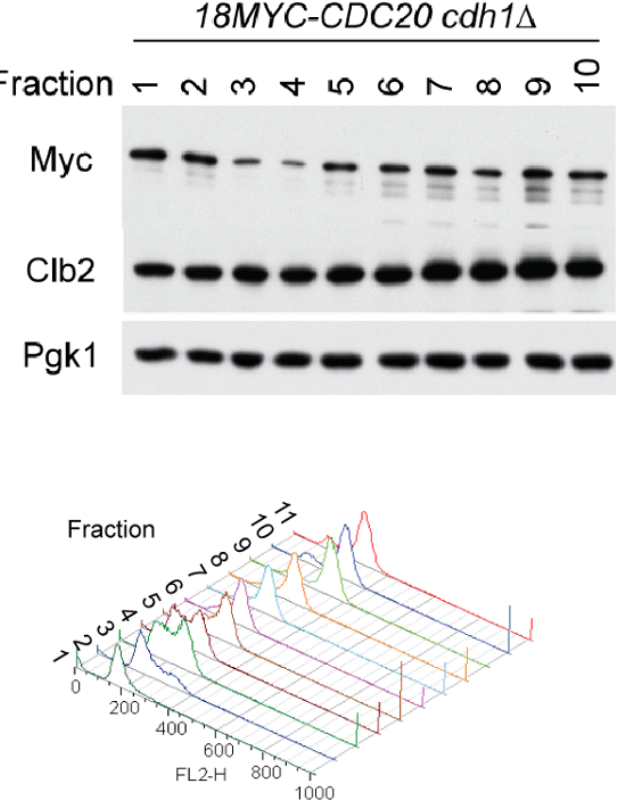

Figure 4 Deletion of $\mathrm{CDH} 1$ partially stabilizes Cdc20. A CDH1 18MYC-CDC20 and cdh1 18MYC-CDC20 strains were elutriated, and the resultant fractions were immunoblotted for Myc and Clb2. Pgk1 serves as a loading control. B FACS to assess DNA content for the fractions collected in (A). 


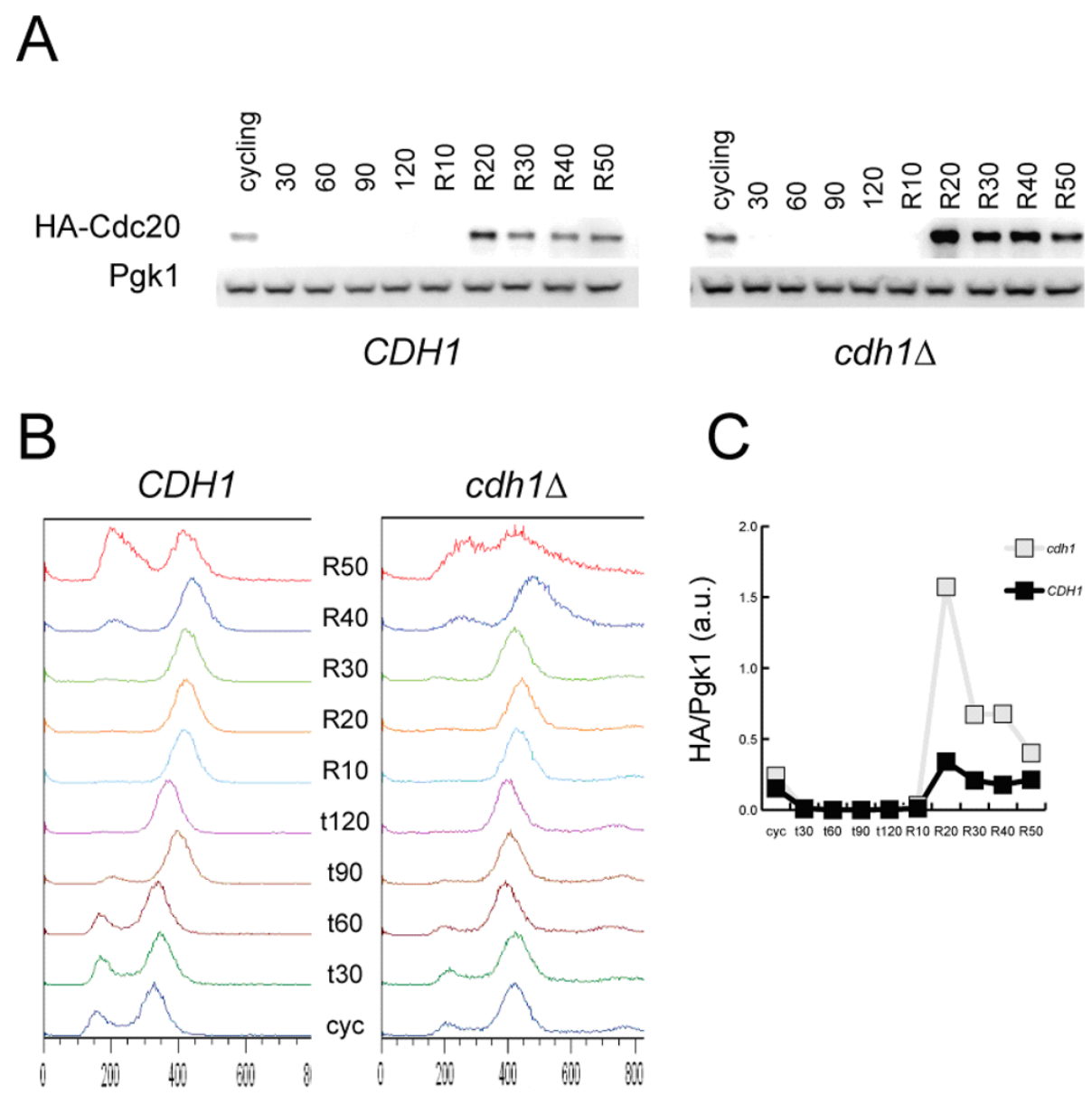

Figure 5 Inducible Cdc20 is degraded by both Cdh1 dependent and independent mechanisms. A Methionine was added at time 0 to cycling CDH1 and cdh1 strains, with MET3pr-HA-CDC20 (methionine repressible) replacing endogenous CDC20, to shutoff CDC20 transcription. After two hours, these cells were released into methionine-free medium, inducing CDC20 transcription. Samples were taken every ten minutes thereafter (R10, R20, etc). HA-Cdc20 was immunoblotted against, with Pgk1 serving as a loading control. B DNA content of the strains in (A) was assessed by FACS. C Quantification of immunoblots in (A).

\section{Discussion}

The regulatory control of Cdc20 has implications for the proper ordering of cell cycle events. Multiple mechanisms are involved in its regulation including transcriptional control, Cdc20 protein destruction, and CDK phosphorylation of the APC. Both CDC20 transcriptional control and CDK phosphorylation of Cdc16, Cdc23, and Cdc27 APC components are dispensable for cell cycle progression $[13,14]$. Cdc20 proteolysis provides a third control mechanism, but the literature is unclear as to how Cdc 20 proteolysis is controlled. In this work, we attempt to clarify the agents, timing and motifs involved in $\mathrm{Cdc} 20$ destruction.

Conflicting reports exist in the literature as to what mediates the destruction of Cdc20. Studies have variously implicated the APC, probably independent of Cdh1 because of the timing of proteolysis $[16,17]$, APCCdh1 specifically [22], and an APC-independent mechanism [30]. Here we find evidence for both APCCdh1 dependent and independent mechanisms, operating at different times in the cell cycle; the existence of multiple mechanisms may account for the conflicting nature of previous reports. One caveat to our experiments are the use of epitope tags, for which there always exists a potential for tag artifacts to occur. However, we find consistent evidence for APC dependent and independent mechanisms of Cdc20 degradation using both Myc and HA tags, arguing against the presence of such an artifact. Additionally, the epitope tags have the advantage of precluding the possibility that the antibody is affected by either posttranslational modifications or constructed mutations of Cdc20 (such as destruction box ablation-we have found that the destruction box and KEN boxes of the Clb2 mitotic cyclin can be major epitopes for rabbit antibodies, for example [B. Drapkin, pers. comm.]). 
We find that APC-Cdh1 makes the major contribution to $\mathrm{Cdc} 20$ proteolysis from mitotic exit through the subsequent G1 (Figure 4). This provides a simple mechanism for temporal separation of APC-Cdc20 and APC-Cdh1 activity: as Cdh1 is activated in late mitosis, Cdc20 is effectively removed by Cdh1-dependent proteolysis. Beginning in late G1/early S, a Cdh1independent mechanism can also carry out Cdc20 degradation. We do not know what proteins control this mechanism, preventing further analysis. The efficiency of removal of destruction box-containing Cdc20 by Cdh1 suggests that this second mechanism is not of great importance for regulating Cdc20 levels. We speculate that this may play role in limiting Cdc20 accumulation prior to anaphase so as to prevent premature cohesin cleavage, or may contribute to efficient engagement of the spindle checkpoint during aberrant mitoses (since Cdc20 is the ultimate target of this checkpoint).

APC-dependent but destruction box independent degradation of Cdc20 was reported in S phase and mitosis [17]; it is possible that this is the Cdh1-and destruction box-independent $\mathrm{Cdc} 20$ degradation we observe. Cdh1 would not be expected to be active in these phases of the cell cycle, and thus any APC-mediated degradation of $\mathrm{Cdc} 20$ would likely result from a direct APC Cdc20 interaction (especially as characterized APC-Cdc20 substrates are stable at this time). We have not evaluated the role of APC-dependent but coactivator-independent ubiquitination of $\mathrm{Cdc} 20$.

Work based upon overexpression studies of $C D C 20$ alleles with destruction box deletions has arrived at conflicting conclusions for the relative contributions of the two destruction boxes $[16,17,30]$. We find both destruction boxes to contribute to Cdc20 instability, particularly during G1. However, the stabilization conferred by destruction box ablation appears to be considerably weaker than the stabilization due to $C D H 1$ removal. This suggests that APC-Cdh1 mediates the destruction of Cdc20 through both destruction box dependent and independent mechanisms. This destruction box independent mechanism could either be through direct degradation or an indirect mechanism such as altering synthesis or localization. There could be additional APC-Cdh1targeting motifs in $\mathrm{Cdc} 20$; one possible targeting motif is a potential KEN box in the C-terminal portion of Cdc20. It is also possible that Cdh1 affects Cdc20 transcription, perhaps by targeting $\mathrm{Clb} 2$ which promotes CDC20 transcription [35,36].

\section{Conclusions}

Cdc20 proteolysis is under complex control, with different systems operating at different points in the cell cycle. This complexity is likely to explain apparent conflicts in previously published literature on this subject. A major mode of control of Cdc20 proteolysis occurs in late mitosis/early G1 and is Cdh1-dependent, as in animal cells; this mode may contribute to the known sequential activation of the APC by Cdc20 followed by Cdh1. An independent mode of $\mathrm{Cdc} 20$ proteolysis, independent of destruction boxes and Cdh1, occurs at G1/S; we do not know the mechanism or function of this mode of proteolysis, but speculate that it may contribute to sharpening and restricting activation of Cdc20-APC to early mitosis.

\section{Methods}

\section{Yeast strains and plasmids}

Standard methods were used throughout. All strains are W303. See Table 1 for strains used. See Table 2 for plasmids used. $C D C 20$ mutagenesis was performed by Quickchange Multi-Site Directed Mutagenesis (Stratagene) using the following primers:

Cdc20-db1: AATGCAGCAATTAGCGGTAACgcTT CTGTAgcTTCTATTGCGTCCCCAACAAAGC (creating R17A and L20A)

Cdc20-db2: CTGAACATTAGAAACTCCAAAgcTCCCAGTgcACAAGCCTCTGCCAATTCTATT (creating (R60A and L63A)

\section{Synchronization}

Alpha-factor time courses were performed by blocking for between two and three hours in alpha-factor, washing $3 \times$ in alpha-factor-free medium, and releasing into the indicated culture conditions.

MET3-HA-CDC2O time courses were performed by adding $0.2 \mathrm{~g} / \mathrm{L}$ methionine to methionine-free synthetic medium, arresting for between two and three hours, filtering onto nitrocellulose membranes, washing, and releasing into methionine-free medium.

Centrifugal elutriations were performed using $1 \mathrm{~L}$ of log phase culture with a Beckman JE5.0 elutriator rotor, running at 3000RPM with sequential fractions elutriated off by stepwise increase in pump speed.

\section{Table 1 Strains used in this study}

\begin{tabular}{ll}
\hline $\begin{array}{l}\text { Strain } \\
\text { Name }\end{array}$ & Genotype \\
\hline JR90 & MATa TRP1-18MYC-CDC20-WT ADE2 URA3::Ura3 pRS313:GAL4- \\
& MR-HIS3 \\
\hline JR91 & MATa TRP1-18MYC-CDC20-WT ADE2 GALL-HA-CDH1-WT- \\
& URA3::Ura3 pRS313:GAL4-MR-HIS3 \\
\hline JR13 & MATa HIS3::GFP-TUB1 MYO1-GFP-KANMX bar1 MYC-CDC20- \\
& TRP1 ADE2 \\
\hline JR52 & MATa HIS3::GFP-TUB1 MYO1-GFP-KAN bar1 TRP1-18MYC- \\
& CDC20-db1 ADE2 \\
\hline JR55 & MATa HIS3::GFP-TUB1 MYO1-GFP-KANMX bar1 MYC-CDC20- \\
& db2-TRP1 ADE2 \\
\hline JR53 & MATa HIS3::GFP-TUB1 MYO1-GFP-KAN bar1 TRP1-18MYC- \\
& CDC20-db3 ADE2 \\
\hline
\end{tabular}


Table 2 Plasmids used in this study

\begin{tabular}{lll}
\hline $\begin{array}{l}\text { Plasmid } \\
\text { Name }\end{array}$ & Description & Plasmid Notes \\
\hline DJC235 & 18MYC-CDC20-TRP1 & $\begin{array}{l}\text { cut with Mlul integrate at } \\
\text { CDC20 }\end{array}$ \\
\hline JRP1 & 18MYC-CDC20-db1-TRP1 & $\begin{array}{l}\text { cut with Mlul to integrate at } \\
\text { CDC20 }\end{array}$ \\
\hline JRP2 & 18MYC-CDC20-db2-TRP1 & $\begin{array}{l}\text { cut with Mlul to integrate at } \\
\text { CDC20 }\end{array}$ \\
\hline JRP3 & 18MYC-CDC20-db3-TRP1 & $\begin{array}{l}\text { cut with Mlul to integrate at } \\
\text { CDC20 }\end{array}$ \\
\hline JRP5 & pRS406 GALL-HA-CDH1 & $\begin{array}{l}\text { cut with Bglll to integrate at } \\
\text { CDH1 }\end{array}$ \\
\hline JRP6 & pRS406 GALL-HA-CDH1-m11 & $\begin{array}{l}\text { cut with Bglll to integrate at } \\
\text { CDH1 }\end{array}$ \\
\hline
\end{tabular}

\section{Immunoblots}

Western Blots were performed using standard methods. Antibody concentrations used were: anti-Pgk1 1:10,000 (Invitrogen), anti-HA 12CA5 1:1,000 (Roche), rabbit polyclonal anti-Clb2 1:10,000, Myc 9E10 1:1,000 (Santa Cruz Biotechnology), Clb5 yN-19 (Santa Cruz), Cdc5 yC-19 (Santa Cruz), and HRP-conjugated secondary antibodies at 1:4,000. ECL signal was measured in a Fujifilm DarkBox with CCD camera, and quantified using MultiGauge software (Fujifilm).

\section{Flow Cytometry}

DNA content was assessed through propidium iodide staining of ribonuclease treated cells on a FACSCalibur machine (BD Biosciences), as described [37].

\section{Acknowledgements}

We thank N. Buchler, K. Nasmyth, M. Schwab for plasmids, and Lea Schroeder for technical assistance. This work was supported by PHS GM47238. J.A.R. was supported by NIH MSTP grant GM07739.

\section{Authors' contributions}

JAR and FRC designed experiments and drafted the manuscript. JAR performed experiments. Both authors read and approved the final manuscript.

\section{Competing interests}

The authors declare that they have no competing interests.

Received: 5 April 2010 Accepted: 10 September 2010 Published: 10 September 2010

\section{References}

1. Draetta G, Luca F, Westendorf J, Brizuela L, Ruderman J, Beach D: Cdc2 protein kinase is complexed with both cyclin A and B: evidence for proteolytic inactivation of MPF. Cell 1989, 56:829-838.

2. Sudakin V, Ganoth D, Dahan A, Heller H, Hershko J, Luca FC, Ruderman JV, Hershko A: The cyclosome, a large complex containing cyclin-selective ubiquitin ligase activity, targets cyclins for destruction at the end of mitosis. Mol Biol Cell 1995, 6:185-197.

3. King RW, Peters JM, Tugendreich S, Rolfe M, Hieter P, Kirschner MW: A $20 S$ complex containing CDC27 and CDC16 catalyzes the mitosisspecific conjugation of ubiquitin to cyclin B. Cell 1995, 81:279-288.
4. Yoon HJ, Feoktistova A, Wolfe BA, Jennings JL, Link AJ, Gould KL: Proteomics analysis identifies new components of the fission and budding yeast anaphase-promoting complexes. Curr Biol 2002, 12:2048-2054

5. Schwab M, Lutum AS, Seufert W: Yeast Hct1 is a regulator of $\mathrm{Clb2}$ cyclin proteolysis. Cell 1997, 90:683-693.

6. Visintin $\mathrm{R}$, Prinz $\mathrm{S}$, Amon $\mathrm{A}: \mathrm{CDC} 20$ and $\mathrm{CDH1}$ : a family of substratespecific activators of APC-dependent proteolysis. Science 1997, 278:460-463.

7. Zachariae W, Shevchenko A, Andrews PD, Ciosk R, Galova M, Stark MJ, Mann M, Nasmyth K: Mass spectrometric analysis of the anaphasepromoting complex from yeast: identification of a subunit related to cullins. Science 1998, 279:1216-1219.

8. Thornton BR, Ng TM, Matyskiela ME, Carroll CW, Morgan DO, Toczyski DP: An architectural map of the anaphase-promoting complex. Genes Dev 2006, 20:449-460.

9. Irniger $\mathrm{S}$, Nasmyth $\mathrm{K}$ : The anaphase-promoting complex is required in G1 arrested yeast cells to inhibit B-type cyclin accumulation and to prevent uncontrolled entry into S-phase. J Cell Sci 1997, 110(Pt 13):1523-1531.

10. Zachariae W, Schwab M, Nasmyth K, Seufert W: Control of cyclin ubiquitination by CDK-regulated binding of Hct1 to the anaphase promoting complex. Science 1998, 282:1721-1724.

11. Shirayama M, Toth A, Galova M, Nasmyth K: APC(Cdc20) promotes exit from mitosis by destroying the anaphase inhibitor Pds1 and cyclin Clb5. Nature 1999, 402:203-207.

12. Wäsch R, Cross FR: APC-dependent proteolysis of the mitotic cyclin Clb2 is essential for mitotic exit. Nature 2002, 418:556-562.

13. Yeong FM, Lim HH, Padmashree CG, Surana U: Exit from mitosis in budding yeast: biphasic inactivation of the $\mathrm{Cdc} 28-\mathrm{Clb} 2$ mitotic kinase and the role of Cdc20. Mol Cell 2000, 5:501-511.

14. Rudner AD, Murray AW: Phosphorylation by Cdc28 activates the Cdc20dependent activity of the anaphase-promoting complex. J Cell Biol 2000, 149:1377-1390.

15. Kramer ER, Scheuringer N, Podtelejnikov AV, Mann M, Peters JM: Mitotic regulation of the APC activator proteins $\mathrm{CDC} 20$ and $\mathrm{CDH} 1$. Mol Biol Cell 2000, 11:1555-1569.

16. Shirayama M, Zachariae W, Ciosk R, Nasmyth K: The Polo-like kinase Cdc5p and the WD-repeat protein Cdc20p/fizzy are regulators and substrates of the anaphase promoting complex in Saccharomyces cerevisiae. Embo J 1998, 17:1336-1349.

17. Prinz $S$, Hwang ES, Visintin $R$, Amon A: The regulation of $C d c 20$ proteolysis reveals a role for APC components $\mathrm{Cdc} 23$ and $\mathrm{Cdc} 27$ during S phase and early mitosis. Curr Biol 1998, 8:750-760.

18. Weinstein J: Cell cycle-regulated expression, phosphorylation, and degradation of p55Cdc. A mammalian homolog of CDC20/Fizzy/slp1. J Biol Chem 1997, 272:28501-28511.

19. Amon A, Irniger $S$, Nasmyth $K$ : Closing the cell cycle circle in yeast: $G 2$ cyclin proteolysis initiated at mitosis persists until the activation of $\mathrm{G} 1$ cyclins in the next cycle. Cell 1994, 77:1037-1050.

20. Cohen-Fix O, Peters JM, Kirschner MW, Koshland D: Anaphase initiation in Saccharomyces cerevisiae is controlled by the APC-dependent degradation of the anaphase inhibitor Pds1p. Genes Dev 1996, 10:3081-3093.

21. Hildebrandt ER, Hoyt MA: Cell cycle-dependent degradation of the Saccharomyces cerevisiae spindle motor Cin8p requires $\mathrm{APC}(\mathrm{Cdh} 1)$ and a bipartite destruction sequence. Mol Biol Cell 2001, 12:3402-3416.

22. Huang JN, Park I, Ellingson E, Littlepage LE, Pellman D: Activity of the APC (Cdh1) form of the anaphase-promoting complex persists until $S$ phase and prevents the premature expression of Cdc20p. J Cell Bio/ 2001, 154:85-94.

23. Juang YL, Huang J, Peters JM, McLaughlin ME, Tai CY, Pellman D: APCmediated proteolysis of Ase1 and the morphogenesis of the mitotic spindle. Science 1997, 275:1311-1314.

24. Woodbury EL, Morgan DO: Cdk and APC activities limit the spindlestabilizing function of Fin1 to anaphase. Nat Cell Biol 2007, 9:106-112.

25. King RW, Glotzer M, Kirschner MW: Mutagenic analysis of the destruction signal of mitotic cyclins and structural characterization of ubiquitinated intermediates. Mol Biol Cell 1996, 7:1343-1357.

26. Pfleger CM, Lee E, Kirschner MW: Substrate recognition by the Cdc20 and Cdh1 components of the anaphase-promoting complex. Genes Dev 2001, 15:2396-2407. 
27. Burton JL, Solomon MJ: D box and KEN box motifs in budding yeast Hsl1p are required for APC-mediated degradation and direct binding to Cdc20p and Cdh1p. Genes Dev 2001, 15:2381-2395.

28. Reis $A$, Levasseur M, Chang HY, Elliott DJ, Jones KT: The CRY box: a second APCcdh1-dependent degron in mammalian cdc20. EMBO Rep 2006, 7:1040-1045.

29. Sethi N, Monteagudo MC, Koshland D, Hogan E, Burke DJ: The CDC20 gene product of Saccharomyces cerevisiae, a beta-transducin homolog, is required for a subset of microtubule-dependent cellular processes. Mol Cell Biol 1991, 11:5592-5602.

30. Goh PY, Lim HH, Surana U: Cdc20 protein contains a destruction-box but, unlike Clb2, its proteolysisis not acutely dependent on the activity of anaphase-promoting complex. Eur J Biochem 2000, 267:434-449.

31. Cross FR: Two redundant oscillatory mechanisms in the yeast cell cycle. Dev Cell 2003, 4:741-752.

32. Spellman PT, Sherlock G, Zhang MQ, lyer VR, Anders K, Eisen MB, Brown PO, Botstein D, Futcher B: Comprehensive identification of cell cycleregulated genes of the yeast Saccharomyces cerevisiae by microarray hybridization. Mol Biol Cell 1998, 9:3273-3297.

33. Robbins JA, Cross FR: Requirements and reasons for effective inhibition of the anaphase promoting complex activator CDH1. Mol Biol Cell 21:914-925.

34. Cross FR, Archambault V, Miller M, Klovstad M: Testing a mathematical model of the yeast cell cycle. Mol Biol Cell 2002, 13:52-70.

35. Amon A, Tyers M, Futcher B, Nasmyth K: Mechanisms that help the yeast cell cycle clock tick: G2 cyclins transcriptionally activate G2 cyclins and repress G1 cyclins. Cell 1993, 74:993-1007.

36. Zhu G, Spellman PT, Volpe T, Brown PO, Botstein D, Davis TN, Futcher B: Two yeast forkhead genes regulate the cell cycle and pseudohyphal growth. Nature 2000, 406:90-94.

37. Epstein CB, Cross FR: CLB5: a novel B cyclin from budding yeast with a role in S phase. Genes Dev 1992, 6:1695-1706.

38. Mattioni T, Louvion JF, D P: Regulation of protein activities by fusion to steroid binding domains. Methods Cell Biol 1994, 43(Pt A):335-352.

doi:10.1186/1747-1028-5-23

Cite this article as: Robbins and Cross: Regulated degradation of the APC coactivator Cdc20. Cell Division 2010 5:23.

\section{Submit your next manuscript to BioMed Central and take full advantage of:}

- Convenient online submission

- Thorough peer review

- No space constraints or color figure charges

- Immediate publication on acceptance

- Inclusion in PubMed, CAS, Scopus and Google Scholar

- Research which is freely available for redistribution

Submit your manuscript at www.biomedcentral.com/submit
Biomed Central 